\title{
Socio-demographic correlates of wildlife consumption during early stages of the COVID-19 pandemic
}

\author{
Robin Naidoo ${ }^{1,2} \bowtie$, Daniel Bergin ${ }^{3,4}$ and Jan Vertefeuille ${ }^{1}$
}

\begin{abstract}
To inform efforts at preventing future pandemics, we assessed how socio-demographic attributes correlated with wildlife consumption as COVID-19 (coronavirus disease 2019) first spread across Asia. Self-reported wildlife consumption was most strongly related to COVID-19 awareness; those with greater awareness were $\mathbf{1 1 - 2 4 \%}$ less likely to buy wildlife products. A hypothetical intervention targeting increased awareness, support for wildlife market closures and reduced medical impacts of COVID-19 could halve future wildlife consumption rates across several countries and demographics.
\end{abstract}

The global COVID-19 pandemic has killed over four million people around the world and caused trillions of dollars of economic damage, but it did not arise unexpectedly. Indeed, experts had warned of this type of large-scale outbreak in the wake of other recent emerging zoonotic diseases ${ }^{1}$. While uncertainty remains regarding the specific origin of COVID- $19^{2}$, a key driving force of emerging infectious diseases of zoonotic origin is the trade and consumption of wildlife, in particular of high-risk taxa ${ }^{3}$, or of species sold in high-risk market conditions ${ }^{4}$. While the global costs of pandemics such as COVID-19 drastically exceed the benefits of the global wildlife trade ${ }^{5}$, it has nevertheless proven difficult to address large-scale wildlife consumption at local or regional scales. This is especially true in certain Asian countries where demand for wildlife used in various traditional, cultural and economic contexts is high ${ }^{6}$, and where attempts to curb illegal trade are sometimes hampered by weak wildlife trade laws, low enforcement rates and/or corruption?

The global conservation community is debating the best long-term response to COVID-19, in particular on how to reduce wildlife consumption and habitat destruction so that the probability of future pandemic emergence is reduced ${ }^{8-10}$. Regulatory approaches such as the closing of wildlife markets-especially those deemed high-risk-are a popular demand ${ }^{8}$; however, previous examples have shown that rendering the consumption of certain goods illegal (for example, alcohol, recreational drugs) can drive existing demand underground to black markets ${ }^{11}$. Closing markets or otherwise restricting access to wildlife in situations where trade is highly localized, and/or where wildlife use is imperative for livelihoods or subsistence, also poses ethical dilemmas and trade-offs that are not easily answered ${ }^{8,12}$.

A complement to regulatory approaches are demand reduction efforts, which seek to influence consumer preferences so that demand for wildlife is reduced, leading to lower consumption rates. Reducing consumer demand may be a more comprehensive approach to lessening wildlife consumption ${ }^{13}$, but is beset by many complications, including limited investment in research to understand what drives individuals to consume wildlife ${ }^{14}$. Non-governmental organizations and academics are increasingly cognizant of the need for a solid research foundation to feed into behaviour change campaigns to reduce demand. Recent studies have made advances in identifying motivations for wildlife purchasing, as well as in developing consumer surveys that can help target specific groups of interest rather than whole populations ${ }^{15,16}$. The increasing popularity of demand reduction campaigns ${ }^{13,17}$ can be usefully bolstered by empirical studies that provide evidence-based justification for targeting and messaging strategies ${ }^{18,19}$, which would ultimately allow these interventions to realize their full potential within a comprehensive 'One Health' approach to zoonotic disease regulation ${ }^{20}$.

To address this empirical aspect of wildlife demand reduction efforts, we surveyed a total of 5,000 respondents among the general public in five countries and territories in Asia (Hong Kong SAR, Japan, Myanmar, Thailand and Vietnam), eliciting their self-reported wildlife consumption patterns, their awareness of and attitudes towards wildlife markets and COVID-19, and a variety of socio-demographic information (Methods). We built Bayesian hierarchical regression models on the basis of respondent socio-demographic attributes for (1) self-reported wildlife consumption in the previous 12 months, (2) change in consumption as a result of COVID-19 and (3) anticipated future wildlife consumption (Methods and Fig. 1a). Wildlife consumption in our case referred specifically to the purchase of terrestrial wild animals or their derived products in open, in-country markets such as 'wet' markets (see Supplementary Methods for all questions used in our modelling). We then used insights from these models to develop a simulated behaviour change intervention and assessed the impact this intervention could have on future wildlife consumption.

Our models of recent wildlife-purchasing behaviour and COVID-related changes in wildlife consumption had excellent in-sample goodness-of-fit, with areas under the receiver operating curve, using posterior predictive probability of models, equal to 0.84 and 0.83 , respectively ${ }^{21}$ (Supplementary Fig. 1). The area under the receiver operating curve for the model for future wildlife product purchases was lower at 0.76 , but still at a level considered to provide acceptable classification performance ${ }^{21}$. The model containing all independent variables had the highest predictive power for recent self-reported wildlife consumption, and was statistically indistinguishable from the best reduced-form models for future wildlife consumption and for COVID-related changes in wildlife

'World Wildlife Fund, Washington, DC, USA. ${ }^{2}$ Institute for Resources, Environment and Sustainability, University of British Columbia, Vancouver, British Columbia, Canada. ${ }^{3}$ GlobeScan, Sheung Wan, Hong Kong, SAR, China. ${ }^{4}$ Oxford Wildlife Trade Research Group, Department of Anthropology and Geography, Oxford Brookes University, Oxford, UK. 凶e-mail: robin.naidoo@wwfus.org 
(1) Has anyone you know (e.g. friends, colleagues, family or yourself) bought wildlife products in an open wildlife market in the past 12 months?

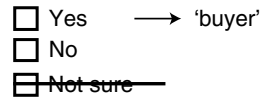

(2) How has the coronavirus affected your consumption of wildlife products?

$\square$ I consume more wildlife products
$\square$ There's no change in my consumption
$\square$ I consume less wildlife products
$\square$ I've completely stopped consuming any wildlife products
$\square$ I never consume wildlife products

(3) How likely will you be to buy wildlife products in open wildlife markets in the future?

$\square$ Very likely
$\square$ Likely
$\square$ Neither likely, nor unlikely
$\square$ Unlikely
$\square$ Very unlikely
'future buyer'

$\square$ Unlikely

c

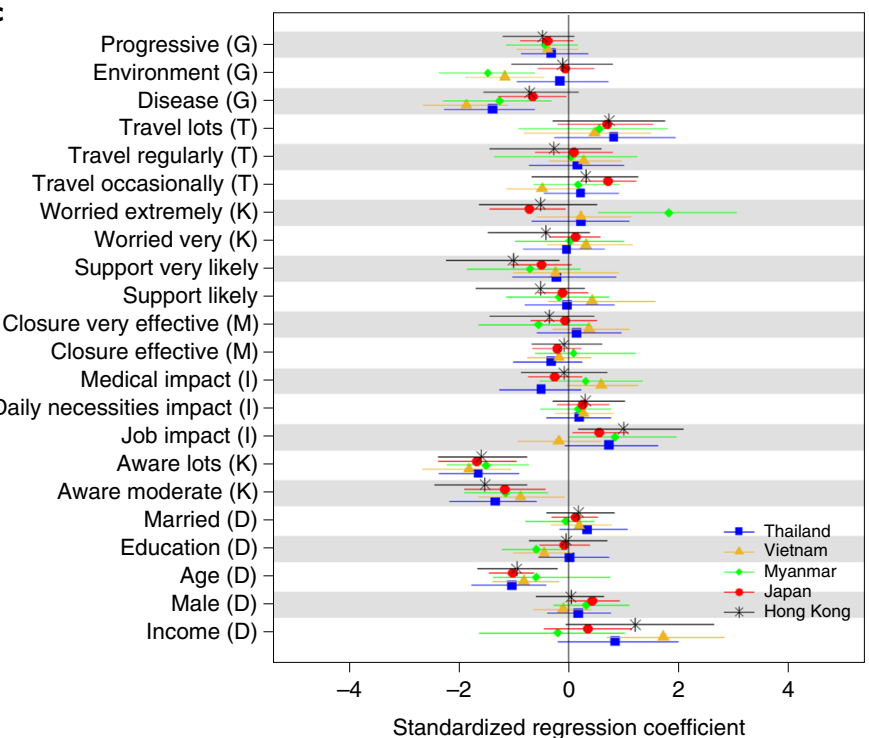

b

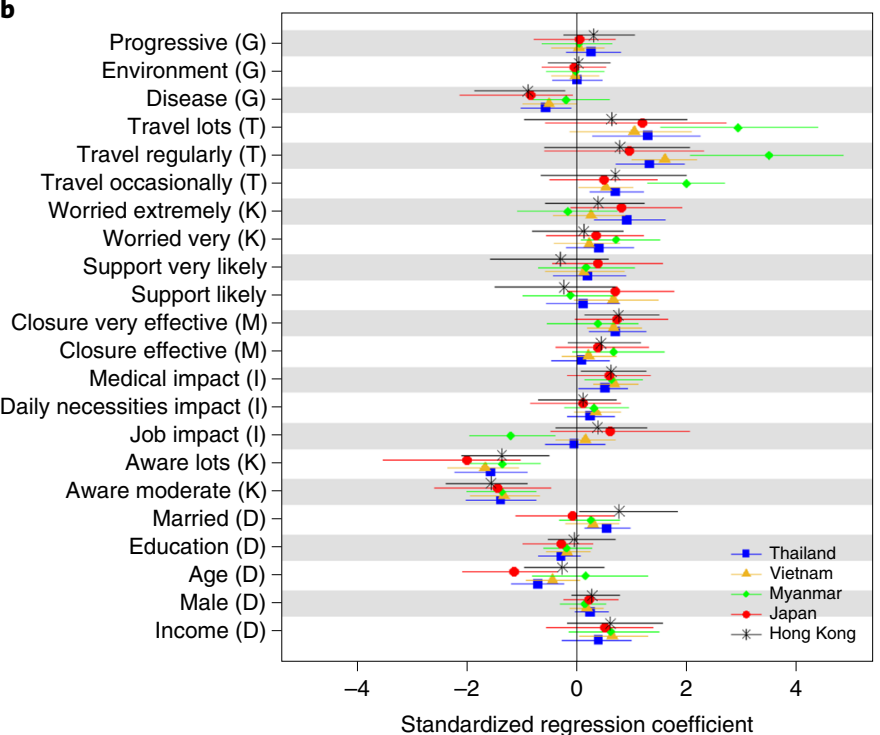

d

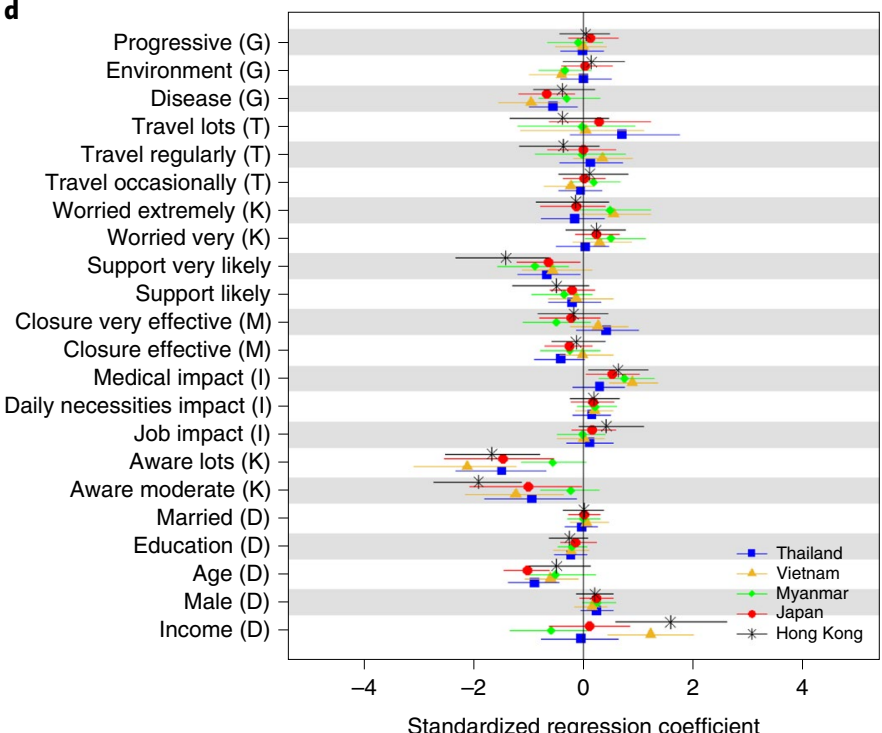

Fig. 1 | Models of wildlife consumption across five Asian territories/countries. a-d, Details of the question responses we modelled (a) and coefficient plots, with $95 \%$ credible intervals, of country-specific estimates from hierarchical Bayesian logistic regression models of questions related to wildlife consumption across five Asian countries/territories, for 'buyer' (b), 'consumption same/increased' (c) and 'future buyer' (d). See Supplementary Table 1 for variable descriptions. Abbreviations in b-d refer to groupings of variables into six categories: G, general attitudes towards global issues; T, international travel habits; K, awareness of COVID-19 and level of worry about future pandemics; D, basic demographics; I, COVID-19 personal impacts; M, support for and effectiveness of wildlife market closures. Note removal of 'Not sure' category for designation of 'buyer'.

consumption (Supplementary Table 2). As has been suggested, we therefore retained the model containing all predictor variables for inference and subsequent predictive modelling across all three response variables ${ }^{22}$.

For all five countries/territories, awareness of COVID-19 was the strongest predictor of whether someone responded positively to any of the three questions regarding self-reported wildlife consumption (that is, current, future and changes as a result of COVID-19; Fig. 1b-d). For all three questions and across all countries/territories, there was strong evidence for negative associations between the highest level of awareness of COVID-19 and the probability of respondents saying they or someone they know would purchase wildlife. There was also strong evidence of a negative association between having some awareness of COVID-19 and the probability of a respondent reporting yes to each consumption question.
The exceptions to this were respondents in Vietnam to the question on changes in wildlife consumption as a result of COVID-19 and in Myanmar to the question on the probability of being a future buyer.

Questions related to potential wildlife market closures had variable associations with wildlife consumption. Respondents in Thailand who viewed wildlife market closures as effective against future pandemics were less likely to say they would consume wildlife in the future. In all countries and territories except Myanmar, respondents who thought wildlife closures would be very effective in preventing future pandemics were actually more likely to have reported wildlife purchases among their social circle in the last 12 months. This may be explained by the fact that the people most familiar with these markets and the conditions wildlife are kept in may also be best placed to understand how closing them may protect public health. Those who were very likely to support government 


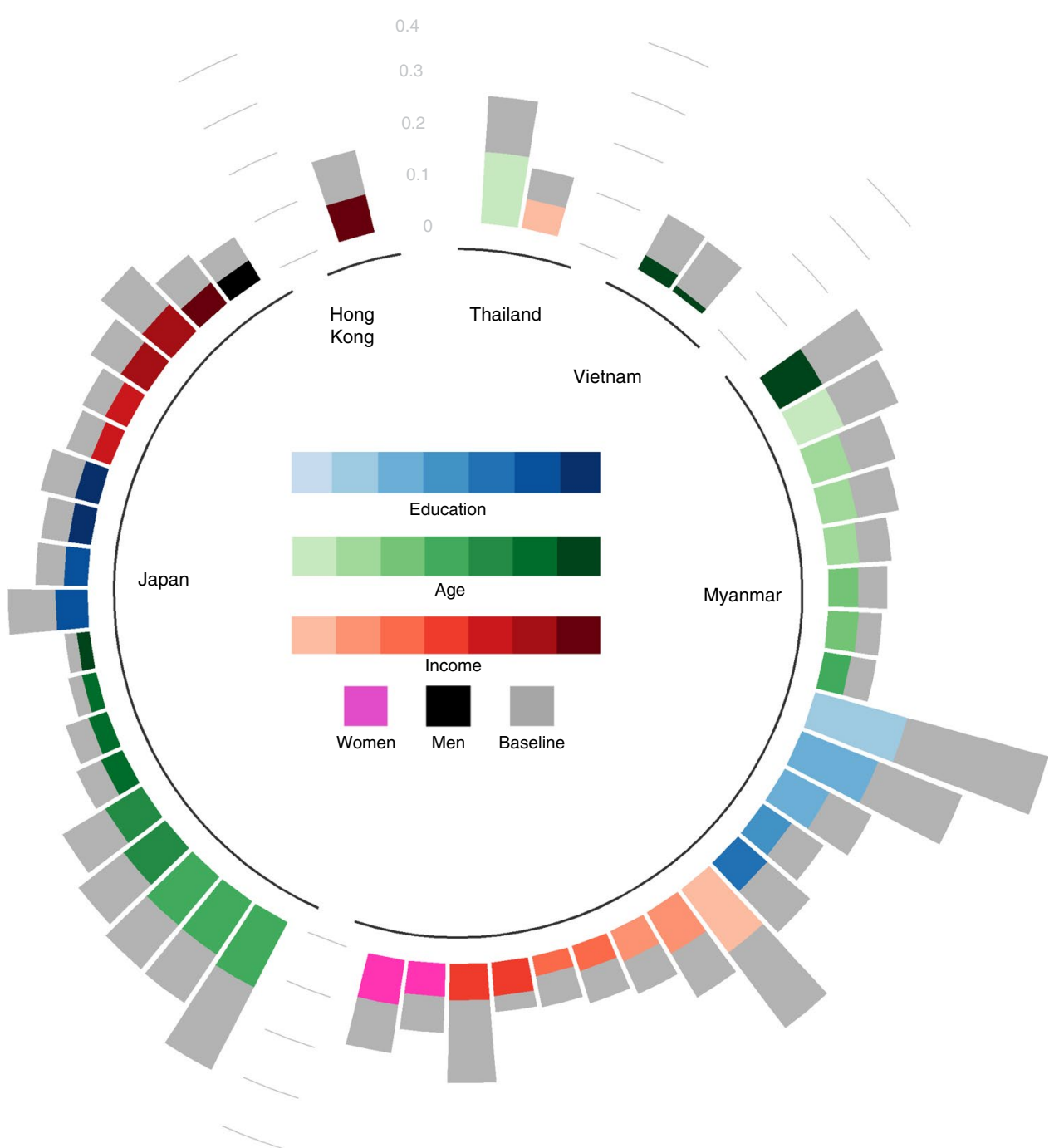

Fig. 2 | Predicted impacts on future wildlife consumption arising from a hypothetical intervention in a target population with the same demographic characteristics as the sample we analysed. Grey bars indicate the mean baseline estimated probability of future wildlife consumption in our target population; coloured bars indicate the mean probability of consumption in various demographic classes after the simulated intervention, with darker shades indicating higher levels of education, age or income. In all cases, these model predictions indicate strong evidence for reductions in self-reported future wildlife consumption (also see Supplementary Table 3, which contains information on Bayesian credible intervals for the differences in mean consumption for baseline versus intervention, for the same countries and demographic classes shown here).

closures of wildlife markets were less likely to say they would consume wildlife in the future in all countries except for Vietnam, where those who were extremely worried about a future pandemic were more likely to have increased their wildlife consumption as a result of COVID-19.

A subset of other demographic and/or attitudinal variables had consistent statistical associations with wildlife-purchasing behaviour. Increased propensity to travel was a strong positive correlate of recent wildlife purchase for respondents in Myanmar (in particular), Thailand and Vietnam. Individuals who rated human disease transmission as the issue that most concerned them were less likely to buy wildlife now or in the future, or to have increased their consumption of wildlife products as a result of COVID-19, across most of the five markets. Younger individuals were consistently associated with wildlife consumption in Thailand, Vietnam and Japan. Income had a strong positive effect on wildlife purchasing in Vietnam, and in the case of future wildlife consumption, in Hong Kong SAR. Across all markets, those who stated the pandemic had strong impacts on their job or livelihoods were more likely to have increased wildlife consumption as a result of COVID-19, while those who stated it had affected their access to medical treatment were more likely to have made recent wildlife purchases, and were more likely to buy again in the future. This latter result is likely because traditional medicines containing wildlife products may act as a surrogate for conventional medical treatment during this time of restricted access to conventional medicine.

We simulated the impacts of a hypothetical intervention package that simultaneously targeted several socio-demographic variables, assessing how future wildlife-purchasing behaviour might change compared to baseline expectations of a population with similar attributes to the one we sampled. The intervention included information provisioning to raise awareness on COVID-19, as well as a hypothetical elimination of medical impacts associated with the pandemic and the achievement of universal support for wildlife market closures. There is strong evidence that this hypothetical intervention would result in substantial reductions in the probability of future buying across simulated populations in Myanmar (mean frequency of future buying reduced from $15.5 \%$ 
to $7.3 \%$ ) and Japan (mean frequency of future buying reduced from $10 \%$ to $4.5 \%)$. There was also strong evidence for reductions in future wildlife consumption among specific demographic groups in all countries/territories (Fig. 2 and Supplementary Table 3). For example, exposing simulated individuals aged $21-25$ in Thailand to the hypothetical intervention resulted in a reduction in the mean probability of future buying from $24.1 \%$ to $13.5 \%$ (a nearly $50 \%$ reduction). And in Hong Kong SAR, our models suggest that targeting wealthier individuals (those earning $>$ US $\$ 135,000$ per year) would reduce the mean probability of future buying in that group from $16 \%$ to $7 \%$ (Fig. 2).

Our results provide clues on how to best approach potential interventions that focus on the demand side of wildlife consumption in parts of Asia, and are particularly relevant for consumption that occurs in high-risk markets where live and/or freshly butchered wildlife and their derived products may be sold for luxury consumption, medicinal use, ornaments or as pets. They show the importance of identifying target groups and target messages before conducting demand reduction campaigns, as results may vary among demographically distinct groups or in different regions. They also suggest areas for follow-up work that should build on the survey we report here. These include further investigation on the drivers of consumer demand for wildlife in Myanmar, Thailand and Vietnam (where consumption levels were highest), as well as surveys in additional countries of importance (for example, China). The opinion poll results we present could also be usefully complemented with experimental survey techniques that address how to elicit information and trade-offs on sensitive topics such as wildlife consumption ${ }^{23-25}$ as well as the psychosocial motivations that may not surface during a traditional survey. Ultimately, basing potential behaviour-change interventions on the best available data and analytical approaches reduces the chance of unintended negative consequences when making policy decisions on wildlife consumption ${ }^{8}$, and could greatly increase the effectiveness and efficiency of these campaigns ${ }^{26}$ within a 'One Health' approach to confronting zoonotic disease emergence.

\section{Methods}

We focused our research on countries/territories in Asia (specifically, Hong Kong SAR, Japan, Myanmar, Thailand and Vietnam) because COVID-19 had not spread much outside Asia at the time of data collection and the global effects were predominantly concentrated in East and Southeast Asia. Our five survey countries/ territories were chosen because they all have relatively high levels of wildlife trade but also represent very different forms of trade (for example, the pet trade in Japan versus the wild-meat trade in Vietnam). Surveying respondents from markets with these different forms of trade thus allowed an examination of how the full variety of wildlife consumption types may be impacted by perceived disease risk. Budgetary constraints precluded the inclusion of further countries, although we believe those that were surveyed provide a valid snapshot of the main regional issues and patterns. The exception to this may be the exclusion of China, a key global player in the wildlife trade and the possible origin of the COVID-19 virus. Conducting research in China requires an extensive process to obtain permission that was not consistent with the opportunistic nature of our survey, which was mobilized quickly to target opinions from a snapshot view of an (at that time) emerging disease. Given the time-sensitive nature of the research, we were therefore unable to wait for the necessary permissions to include China in this survey.

Our online survey was conducted between March 3-11, 2020 and surveyed 1,000 respondents in each of the five target countries/territories. We designed and translated our questionnaires with local experts to ensure questions were culturally appropriate, understandable and relevant. The survey was a quantitative data collection instrument that comprised 32 questions, lasted on average 8 minutes, and respondents were offered an incentive for participating. Respondents aged $18+$ were invited via email from an online panel of over 2.5 million people in the target countries/territories, and could answer on any internet-capable device (for example smartphone, tablet, laptop) at their convenience. Only respondents aged 18 and over were eligible to take the survey, which was entirely voluntary. Any respondents working in advertising, public relations, marketing, market research or media industries were screened out to prevent possible bias. The email invite that was sent to participants did not specify the exact nature of the survey to avoid skewing the participants towards those that believed they know about the topic. Instead, the invite indicated that the questions would be about 'consumption and shopping habits'. The panel is maintained by Toluna (https://tolunacorporate. $\mathrm{com} /$ ), an online data collection group focused on providing high-quality market research data to clients in various business and non-business sectors. Toluna builds and maintains large online consumer panels to collect these data while adhering to stringent global and local guidelines for panel management and data quality, and is a member of the European Society for Opinion and Market Research (https://www. esomar.org).

Toluna respects privacy and is committed to protecting personal data. Their privacy policy (https://tolunacorporate.com/legal/privacy-policy/) provides information on how Toluna collects and processes personal data, explains privacy rights and gives an overview of applicable legislation protecting the handling of personal information. Toluna only uses personal data when the law allows the data to be used.

Respondents were asked demographic questions, and quotas based on the most recent census data for each country/territory were used to ensure the final sample profile was nationally representative of age and gender, except in Myanmar where internet access skewed online panel members to a younger male demographic. Specifically, participants were excluded once quotas on age and gender were filled, and again, participants working in advertising/public relations, marketing research or media were excluded from the survey as we believed these jobs could influence responses. Respondents were asked about societal, economic and environmental concerns, their perception of COVID-19 and their attitudes towards wildlife and wildlife consumption (Supplementary Methods). We also excluded respondents who stated that they were unsure whether they or anyone in their social circle had recently purchased wildlife products $(n=421)$, as well as an additional $n=39$ respondents who were unable to answer survey questions that were later included as covariates in our models.

Because of the potentially sensitive nature of wildlife consumption, we asked about past wildlife purchases indirectly, questioning respondents on whether anyone within their social circle, including themselves, had recently purchased wildlife products. Indirect questions can improve answer rates for questions that people may feel uncomfortable about answering honestly ${ }^{27}$. During the pandemic, respondents may have felt uncomfortable about revealing wildlife purchases, given links between wildlife consumption and COVID-19. Additionally, although most wildlife consumption is legal (with restrictions) in the markets surveyed, some is not, and researchers can be perceived as having interests contrary to that of the respondent. For less-sensitive questions on future wildlife consumption and changes in consumption resulting from COVID-19, we asked respondents for their own response rather than that of their social group.

Previous studies have found a high correlation between an individual's admission of using a wildlife product and their likelihood of being within a network of individuals who buy such products ${ }^{28}$, and suggested that this is linked to homophily in social networks, especially in Southeast Asia. The homophily principle states that people's personal networks are homogeneous with regard to many socio-demographic, behavioural and intrapersonal characteristics ${ }^{29}$. Research on wildlife consumption in other Southeast Asian contexts suggests that social groups can be a motivator to begin or maintain consumption of wildlife products ${ }^{28,30}$. Our own previous research supports this, indicating a strong correlation between one's own tiger and ivory purchases and knowing someone within one's social circle who has purchased such products. Additionally and recognizing the homophily principle, behaviour change campaigns targeted at social networks rather than individuals per se are likely to achieve better results than non-targeted campaigns. Changing perceptions of acceptability is a key aspect of social marketing and is used in the social mobilization domain of social and behaviour change communications, which has become a popular framework for reducing demand for illegally traded wildlife products ${ }^{31}$. Influencing people within a wildlife consumer's social network may therefore have a higher rate of efficacy than attempting to influence the perceptions of individuals who do not know any consumers of wildlife.

We used hierarchical Bayesian regression models to assess relationships between socio-demographic explanators and our three response variables: (1) self-reported recent wildlife consumption, (2) change in wildlife consumption as a result of COVID-19 and (3) anticipated future wildlife consumption. Explanatory variables included 22 non-collinear variables in six categories: basic demographics, awareness and level of worry of COVID-19, COVID-19 personal impacts, support for and effectiveness of wildlife market closures, international travel habits and general attitudes towards global issues (Supplementary Table 1) Aside from household income (measured in US dollars per year), age (midpoint of year categories from the survey question) and education (ordinal, reflecting increasing level of schooling), all other variables were categorical; those with more than two categories were collapsed into dummy variables. Income, age and education were standardized and included to investigate whether a person's general socio-economic status affects wildlife consumption. General attitudes towards global issues were expected to reflect aspects of respondents' political tendencies, while travel habits were included to test the hypothesis that those who travel internationally more habitually are, and will be, more frequent consumers of wildlife. Questions regarding awareness and impacts of COVID-19, and concern about future disease epidemics, were asked to determine how the pandemic may be shaping wildlife consumption. Finally, support and perceived effectiveness of wildlife market closures were included as predictor variables since this measure has been suggested as a strong policy lever to reduce wildlife consumption. 
The general structure of all three models was as follows:

$$
\begin{gathered}
y_{i j} \sim \operatorname{Bernoulli}\left(\theta_{i j}\right) \\
\operatorname{logit}(\theta)=\alpha+u_{1}+\beta \mathbf{X}+u_{2} \mathbf{Z}
\end{gathered}
$$

This model allowed both coefficients and intercepts to vary across countries (that is, a 'random-slope random-intercept' model). In equation (1), $y_{i j}$ is whether or not individual $i$ in country $j$ reported wildlife consumption, modelled as a Bernoulli trial with probability $\theta_{i j}$. The logit transformation of $\theta$ (equation 2) is a linear function of parameters $\alpha$ and $u_{1}$ (the fixed intercept term and a vector of the country-specific intercept terms, respectively), as well as a vector of fixed regression coefficients $\beta$ and a vector of country-specific regression coefficients $u_{2}$, with $\mathbf{X}$ and $\mathbf{Z}$ being the corresponding design matrices ${ }^{32}$. For $\alpha$ and $\beta$, we used an improper flat prior over the real numbers, while the group level parameters $u_{1}$ and $u_{2}$ were assumed to arise from a multivariate normal distribution with mean 0 and unknown covariance matrix. The covariance matrix was parameterized by a correlation matrix having a Lewandowski-Kurowicka-Joe prior, and a standard deviation with half-Student $t$ prior with three degrees of freedom ${ }^{32}$.

For the three dependent variables, we evaluated the predictive power of a mode containing all 22 variables, as well as six subset models, using Watanabe-Akaike Information Criterion and leave-one-out cross-validation ${ }^{33}$. Each of these six subset models contained all explanatory variables except for those within one of the six categories described above (for example, all explanatory variables except those relating to international travel habits, all explanatory variables except those relating to support for wildlife market closures). We used this model-comparison approach to test whether any of these categories of explanatory variable were more or less important in explaining wildlife consumption; if particular categories of variable are stronger predictors of wildlife consumption, this could help inform where future conservation interventions should focus on. Watanabe-Akaike Information Criterion and leave-one-out cross-validation are both measures of model predictive accuracy (both use log predictive density as the utility function or comparison metric) and have been suggested as useful metrics for Bayesian model selection ${ }^{33}$. We interpreted variable coefficients whose $95 \%$ Bayesian credible intervals did not contain 0 as providing strong evidence for the impact of that variable on the outcome in each of the three models for self-reported wildlife consumption (that is, recent, future and changes due to COVID-19). Models were estimated using the R statistical computing software $\mathrm{e}^{34}$, in particular the package brms $\mathrm{s}^{32}$, with four chains of 1,000 iterations each, a 500-iteration warm-up period, and with successful convergence verified by confirming that R-hat statistical values were less than or equal to 1.01 (ref. ${ }^{22}$ ).

We used the Bayesian hierarchical model of anticipated future wildlife consumption and generated predicted probabilities of future consumption for our sample population (Fig. 2, grey bars). We then predicted future consumption probabilities for a hypothetical behaviour-change intervention (Fig. 2, coloured bars). This intervention was simulated by setting the 'medical impact' variable to zero for all individuals, and by assigning all individuals into the 'aware lots' and 'support very likely' categories for questions related to level of awareness of COVID-19 and level of support for government closure of domestic wildlife markets, respectively. All other variables for individuals were held at the levels recorded in the surveys. We considered the difference between these two predicted probabilities as the impact of the hypothetical behaviour-change intervention, which we examined at the level of the country/territory and within education, age, income and gender demographic classes. Strong evidence for the effectiveness of this hypothetical intervention among countries and demographic classes was suggested where Bayesian credible intervals around the mean predicted difference were less than zero (Supplementary Table 3).

Reporting Summary. Further information on research design is available in the Nature Research Reporting Summary linked to this article.

\section{Data availability}

The data analysed in this study are available via the Open Science Framework at https://osf.io/z8kbd/.

Received: 19 December 2020; Accepted: 29 July 2021; Published online: 6 September 2021

\section{References}

1. Morse, S. S. et al. Prediction and prevention of the next pandemic zoonosis. Lancet 380, 1956-1965 (2012).

2. Mallapaty, S. What's next in the search for COVID's origins. Nature 592, 337-338 (2021).

3. Gibb, R. et al. Zoonotic host diversity increases in human-dominated ecosystems. Nature 584, 398-402 (2020).

4. Aguirre, A. A., Catherina, R., Frye, H. \& Shelley, L. Illicit wildlife trade, wet markets, and COVID-19: preventing future pandemics. World Med. Health Policy 12, 256-265 (2020).
5. Dobson, A. P. et al. Ecology and economics for pandemic prevention. Science 369, 379-381 (2020).

6. Nijman, V. An overview of international wildlife trade from Southeast Asia. Biodivers. Conserv. 19, 1101-1114 (2010).

7. Wyatt, T., Johnson, K., Hunter, L., George, R. \& Gunter, R. Corruption and wildlife trafficking: three case studies involving Asia. Asian J. Criminol. 13, 35-55 (2018).

8. Roe, D. et al. Beyond banning wildlife trade: COVID-19, conservation and development. World Dev. 136, 105121 (2020).

9. Lindsey, P. et al. Conserving Africa's wildlife and wildlands through the COVID-19 crisis and beyond. Nat. Ecol. Evol. 4, 1300-1310 (2020).

10. Hockings, M. et al. Covid-19 and protected and conserved areas. Parks 26, 7-24 (2020).

11. Miron, J. A. \& Zwiebel, J. The economic case against drug prohibition. J. Econ. Perspect. 9, 175-192 (1995).

12. Biggs, D. et al. Breaking the deadlock on ivory. Science 358, 1378-1381 (2017).

13. Sas-Rolfes, M. 't, Challender, D. W. S., Hinsley, A., Veríssimo, D. \& Milner-Gulland, E. J. Illegal wildlife trade: scale, processes, and governance. Annu. Rev. Environ. Resour. 44, 201-228 (2019).

14. Wilkie, D. S. et al. Eating and conserving bushmeat in Africa. Afr. J. Ecol. 54, 402-414 (2016).

15. Bergin, D., Wu, D. \& Meijer, W. Response to "The imaginary 'Asian Super Consumer': A critique of demand reduction campaigns for the illegal wildlife trade". Geoforum 107, 216-219 (2020).

16. Thomas-Walters, L. et al. Motivations for the use and consumption of wildlife products. Conserv. Biol. 35, 483-491 (2021).

17. Greenfield, S. \& Verissimo, D. To what extent is social marketing used in demand reduction campaigns for illegal wildlife products? Insights from elephant ivory and rhino horn. Soc. Mar. Q. 25, 40-54 (2019).

18. Veríssimo, D. \& Wan, A. K. Y. Characterizing efforts to reduce consumer demand for wildlife products. Conserv. Biol. 33, 623-633 (2019).

19. Olmedo, A., Sharif, V. \& Milner-Gulland, E. J. Evaluating the design of behavior change interventions: a case study of rhino horn in Vietnam. Conserv. Lett. 11, e12365 (2018).

20. Cunningham, A. A., Daszak, P. \& Wood, J. L. N. One health, emerging infectious diseases and wildlife: two decades of progress? Philos. Trans. R. Soc. B Biol. Sci. 372, 20160167 (2017).

21. Hosmer, D. W., Lemeshow, S. \& Sturdivant, R. X. Applied Logistic Regression 3rd edn (John Wiley \& Sons, 2013).

22. Gelman, A. et al. Bayesian Data Analysis 3rd edn (CRC Press, 2013).

23. Nuno, A. \& St John, F. A. V. How to ask sensitive questions in conservation: a review of specialized questioning techniques. Biol. Conserv. 189, 5-15 (2015).

24. Moro, M. et al. An investigation using the choice experiment method into options for reducing illegal bushmeat hunting in western Serengeti. Conserv. Lett. 6, 37-45 (2013).

25. Nuno, A., Bunnefeld, N., Naiman, L. C. \& Milner-Gulland, E. J. A novel approach to assessing the prevalence and drivers of illegal bushmeat hunting in the Serengeti. Conserv. Biol. 27, 1355-1365 (2013).

26. Jacobson, S. K., McDuff, M. D. \& Monroe, M. C. Conservation Education and Outreach Techniques (Oxford Univ. Press, 2015).

27. Bergin, D. \& Nijman, V. in Evolution, Ecology and Conservation of Lorises and Pottos (eds Nekaris, K. \& Burrows, A.) 339-361 (Cambridge Univ. Press, 2020).

28. Davis, E. O., Crudge, B. \& Glikman, J. A. The nominative technique: a simple tool for assessing illegal wildlife consumption. Oryx https://doi.org/10.1017/ S0030605320000745 (2020).

29. McPherson, M., Smith-Lovin, L. \& Cook, J. M. Birds of a feather: homophily in social networks. Annu. Rev. Sociol. 27, 415-444 (2001).

30. Davis, E. O. et al. Understanding the prevalence of bear part consumption in Cambodia: a comparison of specialised questioning techniques. PLoS ONE 14, e0211544 (2019).

31. Burgess, G. Monitoring and Evaluating Behaviour Change Amongst Ilegal Wildlife Product Consumers: Good Practice Guidelines for Social and Behavioural Change Communications Practitioners and Communications Professionals (TRAFFIC, 2018).

32. Burkner, P.-C. brms: an R package for Bayesian multilivel models using Stan. J. Stat. Softw. 80, 1-28 (2017).

33. Vehtari, A., Gelman, A. \& Gabry, J. Practical Bayesian model evaluation using leave-one-out cross-validation and WAIC. Stat. Comput. 27, 1413-1432 (2017).

34. R Core Team. R: A Language and Environment for Statistical Computing ( $\mathrm{R}$ Foundation for Statistical Computing, 2018).

\section{Acknowledgements}

We thank A. Nicolas for research support. 


\section{Author contributions}

J.V. and D.B. conceived the study; D.B. collected the data; R.N. analysed the data; R.N., D.B. and J.V. wrote the paper.

\section{Competing interests}

The authors declare no competing interests.

\section{Additional information}

Supplementary information The online version contains supplementary material available at https://doi.org/10.1038/s41559-021-01546-5.
Correspondence and requests for materials should be addressed to R.N.

Peer review information Nature Ecology \& Evolution thanks Jarno Vanhatalo and the other, anonymous, reviewer(s) for their contribution to the peer review of this work. Peer reviewer reports are available.

Reprints and permissions information is available at www.nature.com/reprints. Publisher's note Springer Nature remains neutral with regard to jurisdictional claims in published maps and institutional affiliations.

(c) The Author(s), under exclusive licence to Springer Nature Limited 2021 


\section{nature portfolio}

Corresponding author(s): Robin Naidoo

Last updated by author(s): Jul 27, 2021

\section{Reporting Summary}

Nature Portfolio wishes to improve the reproducibility of the work that we publish. This form provides structure for consistency and transparency in reporting. For further information on Nature Portfolio policies, see our Editorial Policies and the Editorial Policy Checklist.

\section{Statistics}

For all statistical analyses, confirm that the following items are present in the figure legend, table legend, main text, or Methods section.

n/a Confirmed

$\square$ \. exact sample size $(n)$ for each experimental group/condition, given as a discrete number and unit of measurement

Х $\square$ A statement on whether measurements were taken from distinct samples or whether the same sample was measured repeatedly

$\triangle$ The statistical test(s) used AND whether they are one- or two-sided

$\triangle$ Only common tests should be described solely by name; describe more complex techniques in the Methods section.

$\bigotimes$ A description of all covariates tested

Х $\square$ A description of any assumptions or corrections, such as tests of normality and adjustment for multiple comparisons

$\triangle$ A full description of the statistical parameters including central tendency (e.g. means) or other basic estimates (e.g. regression coefficient)

$\triangle$ AND variation (e.g. standard deviation) or associated estimates of uncertainty (e.g. confidence intervals)

$\triangle \square$ For null hypothesis testing, the test statistic (e.g. $F, t, r$ ) with confidence intervals, effect sizes, degrees of freedom and $P$ value noted

Xive $P$ values as exact values whenever suitable.

$\bigotimes$ For Bayesian analysis, information on the choice of priors and Markov chain Monte Carlo settings

$\bigotimes$ For hierarchical and complex designs, identification of the appropriate level for tests and full reporting of outcomes

Х $\square$ Estimates of effect sizes (e.g. Cohen's d, Pearson's $r$ ), indicating how they were calculated

Our web collection on statistics for biologists contains articles on many of the points above.

\section{Software and code}

Policy information about availability of computer code

Data collection Provide a description of all commercial, open source and custom code used to collect the data in this study, specifying the version used OR state that no software was used.

Data analysis The statistical computing software R (v. 4.0.2) was used for all analyses, in particular package 'brms'.

For manuscripts utilizing custom algorithms or software that are central to the research but not yet described in published literature, software must be made available to editors and reviewers. We strongly encourage code deposition in a community repository (e.g. GitHub). See the Nature Portfolio guidelines for submitting code \& software for further information.

\section{Data}

Policy information about availability of data

All manuscripts must include a data availability statement. This statement should provide the following information, where applicable:

- Accession codes, unique identifiers, or web links for publicly available datasets

- A description of any restrictions on data availability

- For clinical datasets or third party data, please ensure that the statement adheres to our policy 


\section{Field-specific reporting}

Please select the one below that is the best fit for your research. If you are not sure, read the appropriate sections before making your selection.

Life sciences

Behavioural \& social sciences

Ecological, evolutionary \& environmental sciences

For a reference copy of the document with all sections, see nature.com/documents/nr-reporting-summary-flat.pdf

\section{Life sciences study design}

All studies must disclose on these points even when the disclosure is negative.

Sample size Describe how sample size was determined, detailing any statistical methods used to predetermine sample size OR if no sample-size calculation was performed, describe how sample sizes were chosen and provide a rationale for why these sample sizes are sufficient.

Data exclusions Describe any data exclusions. If no data were excluded from the analyses, state so OR if data were excluded, describe the exclusions and the rationale behind them, indicating whether exclusion criteria were pre-established.

Replication Describe the measures taken to verify the reproducibility of the experimental findings. If all attempts at replication were successful, confirm this OR if there are any findings that were not replicated or cannot be reproduced, note this and describe why.

Randomization Describe how samples/organisms/participants were allocated into experimental groups. If allocation was not random, describe how covariates were controlled $O R$ if this is not relevant to your study, explain why.

Blinding

Describe whether the investigators were blinded to group allocation during data collection and/or analysis. If blinding was not possible, describe why OR explain why blinding was not relevant to your study.

\section{Behavioural \& social sciences study design}

All studies must disclose on these points even when the disclosure is negative.

Study description

Research sample

Sampling strategy

Data collection

Timing

Data exclusions

Non-participation

Randomization
We assessed how socio-demographic attributes correlated with wildlife consumption as COVID-19 emerged in Asia. The survey involved a quantitative data collection instrument that comprised 32 questions, lasted on average 8 minutes, and respondents were offered an incentive for participating. We used hierarchical Bayesian regression models of wildlife consumption to predict how a hypothetical behaviour change intervention would change wildlife consumption in a target population with the same characteristics as the one we sampled.

We surveyed 5000 respondents across 5 countries/territories: Hong Kong SAR, Japan, Myanmar, Thailand, and Vietnam.

Respondents aged $18+$ were invited via email from an online panel of over 2.5 million people in the target countries/territory maintained by Toluna. Only respondents aged 18 and over were eligible to take the survey, which was entirely voluntary. The email invite that was sent to participants did not specify the exact nature of the survey to avoid skewing the participants towards those that believed they know about the topic. Instead, the invite indicated that the questions would be about "consumption and shopping habits".

Respondents were invited by email and could answer on any internet-capable device (e.g. smartphone, tablet, laptop) at their convenience.

The survey data were collected from 3 - 11 March 2020, across all 5 countries.

Participants were excluded once quotas on age and gender were filled for each country, and those working in advertising / public relations, marketing research, or media were excluded from the survey as we believed these jobs could influence responses. Postsurvey completion, we excluded survey responses from respondents who were unsure whether they or anyone in their social circle had recently purchased wildlife products $(n=421)$, as well as an additional $n=39$ respondents who were unable to answer survey questions that were included as covariates in our models.

The dropout rate (i.e., those who started the survey and failed to complete it) was $1 \%$. The response rate was more difficult to calculate, because although we know how many email invites were sent across the panel $(n=134,797)$, people are participating on an 'opt-in' basis, and panel members may access surveys in ways other than the email invite (e.g., through links on the panel account website or App). We note also that all types of sample surveys and polls, including ours, may be subject to multiple additional sources of error, including but not limited to sampling error, coverage error, and measurement error.

There was no randomization or experimental grouping of respondents, and the panel maintained by Toluna is not a random sample of national populations. Instead, questions on covariates in 6 categories were asked of all respondents: basic demographics, knowledge of COVID-19, COVID-19 personal impacts, support for and effectiveness of wildlife market closures, level of worry about future pandemics, international travel habits, and general attitudes towards global issues. These were used in subsequent hierarchical Bayesian regression models of wildlife consumption. 


\section{Ecological, evolutionary \& environmental sciences study design}

All studies must disclose on these points even when the disclosure is negative.

Study description We assessed how socio-demographic attributes correlated with wildlife consumption as COVID-19 emerged in Asia. The survey involved a quantitative data collection instrument that comprised 32 questions, lasted on average 8 minutes, and respondents were offered an incentive for participating. We used hierarchical Bayesian regression models of wildlife consumption to predict how a hypothetical behaviour change intervention would change wildlife consumption in a target population with the same characteristics as the one we sampled.

Research sample

We surveyed 5000 respondents across 5 countries/territories: Hong Kong SAR, Japan, Myanmar, Thailand, and Vietnam.

Sampling strategy

We sampled 1000 respondents from each of the 5 countries/territories in our study, which was the maximum sample possible given financial constraints. Respondents aged 18+ were invited via email from an online panel of over 2.5 million people in the target countries/territory maintained by Toluna. Only respondents aged 18 and over were eligible to take the survey. The email invite that was sent to participants did not specify the exact nature of the survey to avoid skewing the participants towards those that believed they know about the topic. Instead, the invite indicated that the questions would be about "consumption and shopping habits".

Data collection $\quad$ Respondents were invited by email and could answer on any internet-capable device (e.g. smartphone, tablet, laptop) at their convenience.

Timing and spatial scale The survey data were collected from 3 - 11 March 2020, across all 5 countries.

Data exclusions Participants were excluded once quotas on age and gender were filled for each country, and those working in advertising / public relations, marketing research, or media were excluded from the survey as we believed these jobs could influence responses. Postsurvey completion, we excluded survey responses from respondents who were unsure whether they or anyone in their social circle had recently purchased wildlife products $(n=421)$, as well as an additional $n=39$ respondents who were unable to answer survey questions that were included as covariates in our models.

Reproducibility

N/A

Randomization

There was no randomization or experimental grouping of respondents, and the panel maintained by Toluna is not a random sample of national populations. Instead, questions on covariates in 6 categories were asked of all respondents: basic demographics, knowledge of COVID-19, COVID-19 personal impacts, support for and effectiveness of wildlife market closures, level of worry about future pandemics, international travel habits, and general attitudes towards global issues. These were used in subsequent hierarchical Bayesian regression models of wildlife consumption.

Blinding

N?A

Did the study involve field work? $\square$ Yes $\$ No

\section{Reporting for specific materials, systems and methods}

We require information from authors about some types of materials, experimental systems and methods used in many studies. Here, indicate whether each material, system or method listed is relevant to your study. If you are not sure if a list item applies to your research, read the appropriate section before selecting a response.
Materials \& experimental systems

\begin{tabular}{l|l}
\hline n/a Involved in the study \\
$\square$ Antibodies \\
$\square$ Eukaryotic cell lines \\
$\square$ Palaeontology and archaeology \\
$\square$ Animals and other organisms \\
$\square$ Human research participants \\
$\square$ Clinical data \\
$\square$ Dual use research of concern
\end{tabular}

Methods $\mathrm{n} / \mathrm{a}$ Involved in the study

$\square$ ChIP-seq

$\square \square$ Flow cytometry

$\square(\square$ MRI-based neuroimaging

\section{Antibodies}

Antibodies used Describe all antibodies used in the study; as applicable, provide supplier name, catalog number, clone name, and lot number.

Validation 


\title{
Eukaryotic cell lines
}

Policy information about cell lines

Cell line source(s)

State the source of each cell line used.

Authentication

Describe the authentication procedures for each cell line used OR declare that none of the cell lines used were authenticated.

Mycoplasma contamination

Confirm that all cell lines tested negative for mycoplasma contamination OR describe the results of the testing for

mycoplasma contamination OR declare that the cell lines were not tested for mycoplasma contamination.

Commonly misidentified lines

(See ICLAC register)

Name any commonly misidentified cell lines used in the study and provide a rationale for their use.

\section{Palaeontology and Archaeology}

Specimen provenance Provide provenance information for specimens and describe permits that were obtained for the work (including the name of the issuing authority, the date of issue, and any identifying information). Permits should encompass collection and, where applicable, export.

Specimen deposition Indicate where the specimens have been deposited to permit free access by other researchers.

Dating methods If new dates are provided, describe how they were obtained (e.g. collection, storage, sample pretreatment and measurement), where they were obtained (i.e. lab name), the calibration program and the protocol for quality assurance OR state that no new dates are provided.

Tick this box to confirm that the raw and calibrated dates are available in the paper or in Supplementary Information.

Ethics oversight

Identify the organization(s) that approved or provided guidance on the study protocol, OR state that no ethical approval or guidance was required and explain why not.

Note that full information on the approval of the study protocol must also be provided in the manuscript.

\section{Animals and other organisms}

Policy information about studies involving animals; ARRIVE guidelines recommended for reporting animal research

\begin{abstract}
Laboratory animals
\end{abstract}
For laboratory animals, report species, strain, sex and age OR state that the study did not involve laboratory animals.

Wild animals

Provide details on animals observed in or captured in the field; report species, sex and age where possible. Describe how animals were caught and transported and what happened to captive animals after the study (if killed, explain why and describe method; if released, say where and when) OR state that the study did not involve wild animals.

Field-collected samples For laboratory work with field-collected samples, describe all relevant parameters such as housing, maintenance, temperature, photoperiod and end-of-experiment protocol OR state that the study did not involve samples collected from the field.

Ethics oversight

Identify the organization(s) that approved or provided guidance on the study protocol, OR state that no ethical approval or guidance was required and explain why not.

Note that full information on the approval of the study protocol must also be provided in the manuscript.

\section{Human research participants}

Policy information about studies involving human research participants

Population characteristics

Populations were nationally representative in all countries except for Myanmar, where the online panel is skewed towards younger men.

Recruitment

Respondents aged $18+$ were invited via email from an online panel of over 2.5 million people in the target countries/territory, maintained by the panel provider Toluna. Respondents were offered an incentive for participating.

Ethics oversight

Identify the organization(s) that approved the study protocol.

Note that full information on the approval of the study protocol must also be provided in the manuscript.

\section{Clinical data}

Policy information about clinical studies

All manuscripts should comply with the ICMJE guidelines for publication of clinical research and a completed CONSORT checklist must be included with all submissions.

Clinical trial registration Provide the trial registration number from ClinicalTrials.gov or an equivalent agency. 


\section{Dual use research of concern}

Policy information about dual use research of concern

\section{Hazards}

Could the accidental, deliberate or reckless misuse of agents or technologies generated in the work, or the application of information presented in the manuscript, pose a threat to:

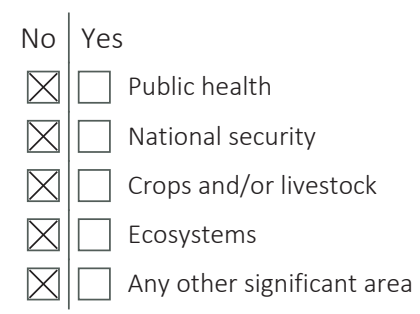

\section{Experiments of concern}

Does the work involve any of these experiments of concern:

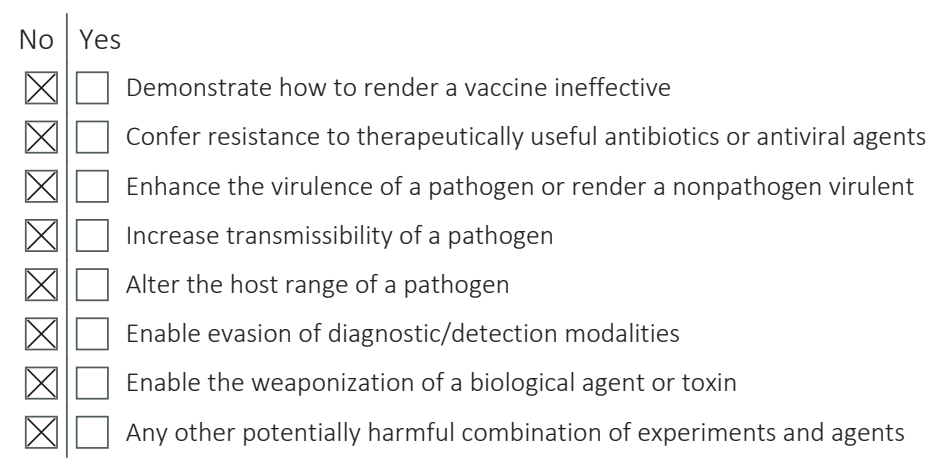

\section{ChIP-seq}

\section{Data deposition}

$\square$ Confirm that both raw and final processed data have been deposited in a public database such as GEO.

Confirm that you have deposited or provided access to graph files (e.g. BED files) for the called peaks.

Data access links

May remain private before publication.

Files in database submission

Genome browser session

(e.g. UCSC)

\section{Methodology}

Replicates

\section{Sequencing depth}

Antibodies

Peak calling parameters
For "Initial submission" or "Revised version" documents, provide reviewer access links. For your "Final submission" document, provide a link to the deposited data.

Provide a list of all files available in the database submission.

Provide a link to an anonymized genome browser session for "Initial submission" and "Revised version" documents only, to enable peer review. Write "no longer applicable" for "Final submission" documents. Describe the experimental replicates, specifying number, type and replicate agreement.

Describe the sequencing depth for each experiment, providing the total number of reads, uniquely mapped reads, length of reads and whether they were paired-or single-end.

Describe the antibodies used for the ChIP-seq experiments; as applicable, provide supplier name, catalog number, clone name, and lot number. 
Data quality

\section{Flow Cytometry}

\section{Plots}

\section{Confirm that:}

$\square$ The axis labels state the marker and fluorochrome used (e.g. CD4-FITC).

$\square$ The axis scales are clearly visible. Include numbers along axes only for bottom left plot of group (a 'group' is an analysis of identical markers).

All plots are contour plots with outliers or pseudocolor plots.

A numerical value for number of cells or percentage (with statistics) is provided

\section{Methodology}

Sample preparation

Instrument

Software

Cell population abundance

Gating strategy
Describe the sample preparation, detailing the biological source of the cells and any tissue processing steps used.

Identify the instrument used for data collection, specifying make and model number.

Describe the software used to collect and analyze the flow cytometry data. For custom code that has been deposited into a community repository, provide accession details.

Describe the abundance of the relevant cell populations within post-sort fractions, providing details on the purity of the samples and how it was determined.

Describe the gating strategy used for all relevant experiments, specifying the preliminary FSC/SSC gates of the starting cell population, indicating where boundaries between "positive" and "negative" staining cell populations are defined.

Tick this box to confirm that a figure exemplifying the gating strategy is provided in the Supplementary Information.

\section{Magnetic resonance imaging}

\section{Experimental design}

Design type

Design specifications

Behavioral performance measures

Acquisition

Imaging type(s)

Field strength

Sequence \& imaging parameters

Area of acquisition

Diffusion MRI

Used
Indicate task or resting state; event-related or block design.

Specify the number of blocks, trials or experimental units per session and/or subject, and specify the length of each trial or block (if trials are blocked) and interval between trials.

State number and/or type of variables recorded (e.g. correct button press, response time) and what statistics were used to establish that the subjects were performing the task as expected (e.g. mean, range, and/or standard deviation across subjects).

\section{Preprocessing}

Preprocessing software

Normalization

Normalization template
Specify: functional, structural, diffusion, perfusion.

Specify in Tesla

Specify the pulse sequence type (gradient echo, spin echo, etc.), imaging type (EPI, spiral, etc.), field of view, matrix size, slice thickness, orientation and TE/TR/flip angle.

State whether a whole brain scan was used OR define the area of acquisition, describing how the region was determined. Not used
Provide detail on software version and revision number and on specific parameters (model/functions, brain extraction, segmentation, smoothing kernel size, etc.).

If data were normalized/standardized, describe the approach(es): specify linear or non-linear and define image types used for transformation OR indicate that data were not normalized and explain rationale for lack of normalization. 
Normalization template

Noise and artifact removal

Volume censoring

Describe your procedure(s) for artifact and structured noise removal, specifying motion parameters, tissue signals and physiological signals (heart rate, respiration).

\section{Statistical modeling \& inference}

Model type and settings

Specify type (mass univariate, multivariate, RSA, predictive, etc.) and describe essential details of the model at the first and second levels (e.g. fixed, random or mixed effects; drift or auto-correlation).

Effect(s) tested

Define precise effect in terms of the task or stimulus conditions instead of psychological concepts and indicate whether ANOVA or factorial designs were used.

Specify type of analysis: ROI-based Both

Statistic type for inference (See Eklund et al. 2016)

Specify voxel-wise or cluster-wise and report all relevant parameters for cluster-wise methods.

Correction

Models \& analysis

$\mathrm{n} / \mathrm{a}$ Involved in the study

$\square \square$ Functional and/or effective connectivity

$\square$ Graph analysis

$\square \square$ Multivariate modeling or predictive analysis

Functional and/or effective connectivity

Graph analysis

Multivariate modeling and predictive analysis
Report the measures of dependence used and the model details (e.g. Pearson correlation, partial correlation, mutual information).

Report the dependent variable and connectivity measure, specifying weighted graph or binarized graph subject-or group-level, and the global and/or node summaries used (e.g. clustering coefficient, efficiency, etc.).

Specify independent variables, features extraction and dimension reduction, model, training and evaluation metrics. 